\title{
PDMS-based Skin-equivalent Phantom for Propagation Studies in the 58-63 GHz Range
}

\author{
A.R. Guraliuc, M. Zhadobov, R. Sauleau \\ Institute of Electronics and Telecommunications of Rennes (IETR), UMR CNRS 6164, University of Rennes 1, Rennes, France \\ anda.guraliuc; maxim.zhadobov; ronan.sauleau@univ-rennes1.fr
}

\begin{abstract}
A solid phantom simulating the same reflection coefficient at the air/phantom interface as at the air/skin interface is developed for on-body propagation assessment in the 58-63 GHz range. The phantom consists in a grounded lossy dielectric sheet made of polydimethylsiloxane (PDMS) and carbon black powder. The paper describes the characteristics of the phantom and provides an example of its application in propagation studies at $60 \mathrm{GHz}$.
\end{abstract}

Keywords: body-centric communications; millimeter-wave propagation; solid phantom.

\section{INTRODUCTION}

In the last years, the interest in millimeter-wave wireless body area networks (WBAN) significantly increased due to some attractive advantages offered by this frequency band. In particular, antennas are more compact compared to those at lower frequencies. Furthermore, this band offers high level of security for short-range communications and reduced interference with neighboring BAN [1-3].

The usefulness of wearable wireless sensors was proven especially in medical applications, where they can replace the wired telemetry systems [4]. For instance, recognizing people's activities is a key issue in assisted living applications [5-6], while patients monitoring or therapy (after an injury, stroke, joint replacement, Parkinson disability) with wearable sensors can significantly improve the health care quality and efficiency. In wearable WBANs a patient wears some sensors like accelerometers, gyroscopes, blood pressure, temperature, ECG sensors, glucose and insulin pump sensor, forming the so called on-body sensor network. In this context, it is important to have a proper propagation channel characteristics in order to establish the on-body network topology, e.g. placement and position of the sensors on the body. Since the human body is an essential component of the propagation scenario, experimental tissue-equivalent phantoms are an efficient tool to test and quantify the wave propagation along and around the human body. They also have the advantage to provide a stable, controllable and reproducible environment for on-body networks testing, which is not easily realized with human subjects.

Various liquid, semi-solid and solid experimental models (phantoms) have been proposed, with dielectric properties simulating the human tissues and covering a wide frequency range from $30 \mathrm{MHz}$ to $10 \mathrm{GHz}$ [7-12], and more recently up to $60 \mathrm{GHz}$ [13]. However, these phantoms present some limitations:

- Liquid phantoms: due to the presence of a container they are not appropriate at millimeter waves (penetration depth at $60 \mathrm{GHz}$ is only about $0.5 \mathrm{~mm} \mathrm{[14]).}$

- Semi-solid (gel) phantoms: due to evaporation dielectric properties of these phantoms degrade over time and their lifetime is typically limited to a few days.

- Solid phantoms: existing solid phantoms do not cover the millimeter-wave band, have a high cost and require specific fabrication conditions.

The purpose of this study is to design a solid dielectric metal-backed phantom for antenna and propagation bodycentric measurements in the $60-\mathrm{GHz}$ band. The phantom model is described in Section II. Its characteristics and an example of its use in on-body propagation studies at $60 \mathrm{GHz}$ are shown in Section III.

\section{Phantom Model}

The millimeter wave - human body interaction is mainly limited to the skin due to the shallow penetration [14]. It has been previously demonstrated that at $60 \mathrm{GHz}$ a homogenous skin-equivalent phantom [13] can be used for on-body propagation characterization [15-17].

For an accurate channel characterization, the reflection coefficient at the air / phantom interface should approach the one at the air/skin interface. The proposed phantom satisfies this requirement; this is achieved by metalizing a thin lossy dielectric sheet which does not necessarily have the same properties as the skin $(\varepsilon=7.98-j \cdot 10.93[18])$. Note that, as a consequence, the absorption inside the phantom could be different with respect to the skin.

Firstly, the dielectric material is realized using polydimethylsiloxane (PDMS - a silicon-based organic polymer) with carbon black powder. Secondly, the homogenous composite is metalized by covering one side with an aluminum foil tape, resulting in a dielectric metal backed phantom (DMBP). A prototype of the DMBP is shown in Fig. 1.

Fig. 2 shows the dielectric properties of the PDMScomposite when carbon black powder is added at different concentrations. These properties have been measured using

This work was supported by Labex CominLabs (French National Research Agency program "Investing for the Future" ANR-10-LABX-0701) and Brittany Region under ResCor/BoWi project, by National Center for Scientific Research (CNRS), and by University of Rennes 1, France. The authors also acknowledge GENCI-IDRIS (Grant 2013-05779). 


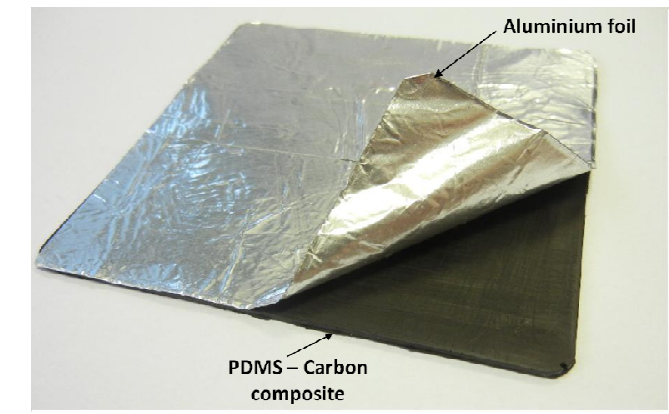

Fig. 1. Phantom prototype.

a free space technique with transmission / reflection quasi optical setup and a millimeter-wave vector network analyzer [19]. It is shown that by increasing the carbon powder concentration up to $60 \%$ (the maximum possible concentration that can be reached due to homogenization issues), the following complex permittivity $\varepsilon=16.23-$ $j \cdot 4.71$ is obtained. It should be noticed that while the real part of the PDMS-composite can be easily reached by increasing the carbon concentration, the imaginary part is more difficult to be obtained (maximum $\operatorname{Im}\{\varepsilon ”\}=4.71$ compared to the target dry skin $\operatorname{Im}\{\varepsilon "\}=10.87$ ).

Since the target reflection coefficient is the one at the air / dry skin [19] interface, the parameters of the DMBP phantom are numerically optimized using the commercial software CST Microwave Studio ${ }^{\circledR}$. A plane wave illuminating a semi-infinite dry skin-equivalent phantom is considered. The reflection coefficient is calculated at $60 \mathrm{GHz}$, for the angle of incidence varying from 0 to $90^{\circ}$ (Fig. 3). Both parallel (TM) and perpendicular (TE) polarizations are considered. It is shown that a DMBP phantom with $\square^{\prime}=11.6, \tan \square=0.25$ (i.e. $\varepsilon=11.6-\mathrm{j} \cdot 2.9$ ) and thickness $h=1.3 \mathrm{~mm}$ provides, at $60 \mathrm{GHz}$, a reflection

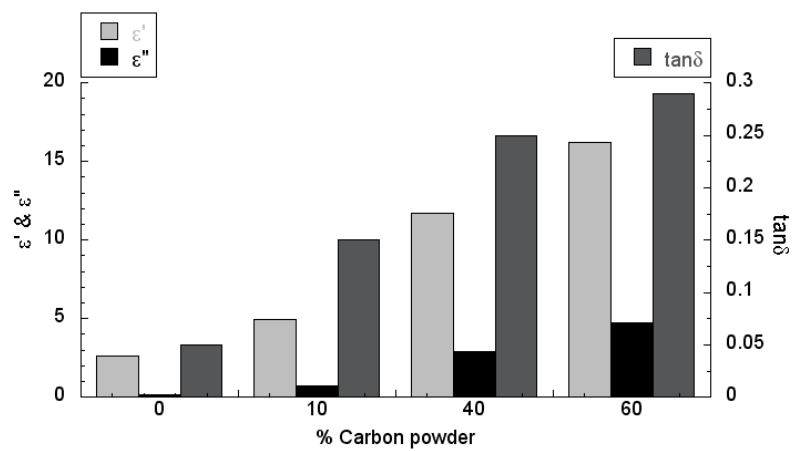

Fig. 2. Measured dielectric properties of the PDMS-carbon powder composite at $60 \mathrm{GHz}$.

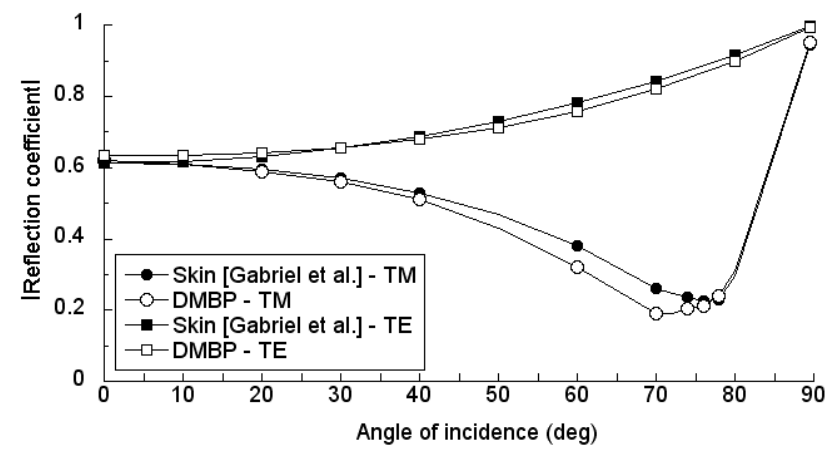

Fig. 3. Power reflection coefficient at $60 \mathrm{GHz}$ : TM (parallel) and TE (perpendicular) polarizations for dry skin and $\operatorname{DMBP}\left(\varepsilon_{r}=11.6\right.$, $\tan \delta=0.25, h=1.3 \mathrm{~mm})$. coefficient at the air / DMBP interface similar to the one at the air / dry skin interface (maximum relative deviation at $70^{\circ}$ is equal to $27 \%$, average deviation is of $6 \%$ for $\mathrm{TM}$ polarization and $3 \%$ for TE polarization).

\section{Characteristics of The Phantom}

In this section, the effects of the DMBP and skinequivalent phantoms on the reflection/transmission coefficients of two waveguide antennas placed above the phantoms are quantified and compared.

At $60 \mathrm{GHz}$, the human body is electrically very large. As a consequence, some body parts can be locally approximated by simple geometrical shapes. For instance the torso can be represented by a rectangular shape. A $30 \times 30 \mathrm{~cm}^{2}$ rectangular DMBP is manufactured [19], as well as a semisolid skin-equivalent phantom with $\varepsilon=8.08-j \cdot 10.87$ [13].

Two standard open-ended waveguide antennas, in Vpolarization, are placed above the phantoms and connected to a Rohde \& Schwarz® ZVA67 VNA using two semi-rigid coaxial cables and waveguide-to-coaxial-cable adapters. Losses in cables and adaptors are taken into account through a full two-port calibration. The measurement setup is schematically shown in Fig. 4.

Fig. 5 shows the reflection coefficient $S_{11}$ and transmission coefficient $S_{2 l}$, when the distance between the waveguides is $15 \mathrm{~cm}$. It can be noticed that the reflection coefficient remains bellow $-10 \mathrm{~dB}$. Also, a very good agreement is shown for the transmission coefficient in the 58-63 GHz range. The small differences (less than $1 \mathrm{~dB}$ ) can be due to a misposition and misalignment of the waveguides.

In the following, an example of the use of the proposed phantom for propagation channel characterization is shown. In particular, the path gain between the two waveguides placed above the phantom is investigated. Fig. 6 shows the measured path gain at $60 \mathrm{GHz}$ versus

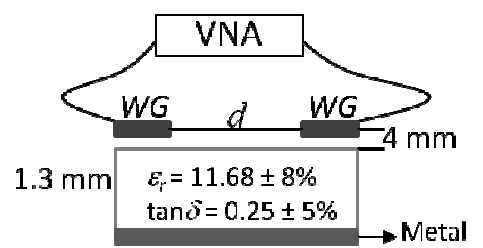

Fig. 4. Two open-ended WR-15 waveguides placed over the surface of the DMBP.

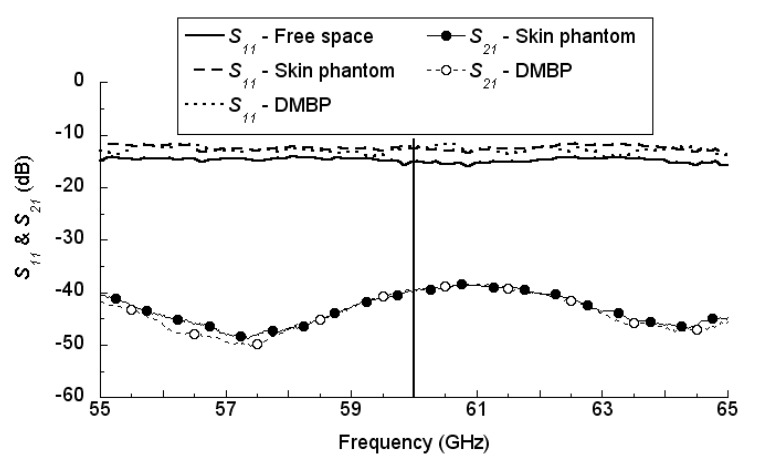

Fig. 5. Measured $S_{11}$ and $S_{21}$ parameters between two open-ended waveguides separated by a distance of $15 \mathrm{~cm}$ and placed above a skinequivalent semi-solid phantom and DMBP. 
waveguides separation distance $d$. A very good agreement is demonstrated between the path gains along the semi-solid skin phantom and along DMBP, respectively. The path gain is characterized by a power decay exponent of around 3.6. This is in agreement with previously reported results [16], [17]. Fig. 7 shows the measured path gain at $58 \mathrm{GHz}$ and 63 $\mathrm{GHz}$ versus separation distance $d$. The results demonstrate a very good agreement between the path gain along the skin and DMBP, further validating the data provided in Fig. 5. A power decay exponent similar to the one at $60 \mathrm{GHz}$ is found at these two frequencies. The path gain has been normalized so that a value of $-25 \mathrm{~dB}$ is found at $d=\lambda_{0}$ on the air / skin interface at $60 \mathrm{GHz}$.

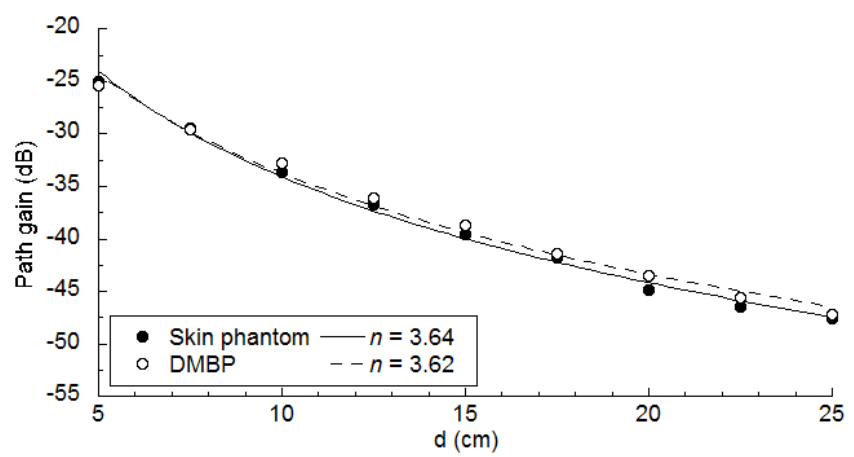

Fig. 6. Measured path gain at $60 \mathrm{GHz}$ versus separation distance $d$ between two open-ended V-polarized waveguides.

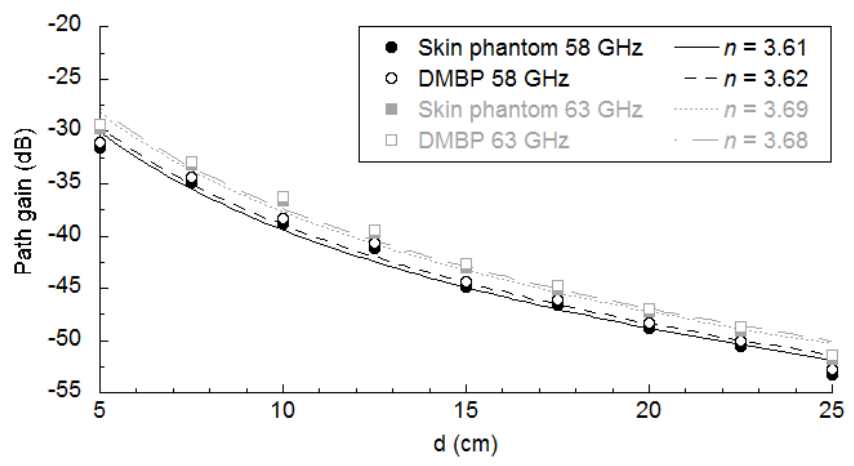

Fig. 7. Measured path gain at $58 \mathrm{GHz}$ and $63 \mathrm{GHz}$ versus separation distance $\mathrm{d}$ between two open-ended V-polarized waveguides.

\section{CONCLUSIONS}

The performances of a dielectric metal-backed phantom for on-body propagation studies at $60 \mathrm{GHz}$ were reported. The proposed phantom simulates the reflection coefficient at the air / phantom interface similar to the one at the air / skin interface. The dielectric material is realized combining PDMS with carbon black powder, and the composite is metalized on one side. The resulting phantom is flexible and simple to fabricate.

Further, as example of its application for on-body propagation, the path gain at $58-60-63 \mathrm{GHz}$ between two open-ended waveguides placed above the DMBP and semisolid skin-equivalent phantom was evaluated. Measured results demonstrated a very good agreement.

The same principle can be applied for designing solid phantoms at other millimeter-wave frequencies.

\section{REFERENCES}

[1] S. L. Cotton, W. G. Scanlon, E. Skafidas, and B. K. Madahar, "Millimeter-wave stealth radio for special operations forces," Microw. J., vol. 53, pp. 6-16, Aug. 2010.

[2] T. Baykas, C. S. Sum, Z. Lan, J. Wang, M. A. Rahman, and H. Harada, "IEEE 802.15.3c: the first IEEE wireless standard for data rates over $1 \mathrm{~Gb} / \mathrm{s}$," IEEE Commun. Mag., vol. 49, no. 7, pp. 114-121, Jul. 2011.

[3] S. L. Cotton, W. G. Scanlon, and P. S. Hall, "A simulated study of cochannel inter-BAN interface at $2.45 \mathrm{GHz}$ and $60 \mathrm{GHz}$," Europ. Wirel. Techn. Conf., Paris, France, pp. 61-64, Sep. 2010.

[4] K. Lorincz, B. Chen, G.W. Challen, A. R. Chowdhury, S. Patel, P. Bonato, and M. Welsh, "Mercury: A wearable sensor network platform for highfidelity motion analysis," Embedded Netw. Sensor Syst. Conf., pp. 183-196, Nov. 2009.

[5] Q. Wang, W. Shin, X. Liu, Z. Zeng, C. Oh, B. K. AlShebli, M. Caccamo, C. A. Gunter, E. Gunter, J. Hou, K. Karahalios, and L. Sha, "I-Living: An open system architecture for assisted living," in Proc. IEEE Int. Conf. Syst., Man Cybern., Oct. 2006, pp. 4268-4275.

[6] M. Annavaram, N. Medvidovic, U. Mitra, S. Narayanan, G. Sukhatme, Z. Meng, S. Qiu, R. Kumar, G. Thatte, and D. SpruijtMetz, "Multimodal sensing for pediatric obesity applications," in Proc. Int. Workshop Urban, Commun., Soc. Appl. Netw. Sens. Syst., Nov. 2008, pp. 21-25.

[7] "MCL-T broadband tissue equivalent liquid: $30 \mathrm{MHz}$ to $6 \mathrm{GHz}$," MCL-T, London, UK [Online]. Available: http://www.mcluk.org/pdfs/bbl.pdf

[8] "IEEE Recommended practice for determining the peak specialaverage specific absorption rate (SAR) in the human head from wireless communication devices: measurement techniques," IEEE Standard 1528-2003, 2003.

[9] K. Ito, K. Furuya, Y. Okano, and L. Hamada, "Development and characteristics of a biological tissue-equivalent phantom for microwaves," Electron. Commun. Japan, vol. 48, no. 4, pp. 67-77, Apr. 2001.

[10] Y. Nikawa, M. Chino, and K. Kikuchi, "Soft and dry phantom modeling material using silicone rubber with carbon fiber," IEEE Microw. Theory Techn., vol. 44, no. 10, pp. 1949-1953, Oct. 1996.

[11] T. Takimoto, T. Onishi, K. Saito, M. Takahashi, S. Uebayashi, and K. Ito, "Characteristics of biological tissue equivalent phantoms applied to UWB communications," Electron. Commun. Japan, vol. 90, no. 5, pp. 48-55, May 2007.

[12] C. Gabriel, "Tissue equivalent material for hand phantoms," Phys. Med. Biol., vol. 53, no. 14, pp. 4205-4210, Jul. 2007.

[13] N. Chahat, M. Zhadobov, and R. Sauleau, "Broadband tissueequivalent phantom for BAN applications at millimeter waves," IEEE Microw. Theory Techn., vol. 60, no. 7, pp. 2259-2266, Jul. 2012.

[14] M. Zhadobov, N. Chahat, R. Sauleau, C. Le Quément, and Y. Le Dréan, "Millimeter-wave interactions with the human body: state of knowledge and recent advances," Int. J. of Microw. and Wirel. Techn., vol. 3, no. 2, pp. 237-247, Apr. 2011.

[15] N. Chahat, G. Valerio, M. Zhadobov, and R. Sauleau, "On-body propagation at $60 \mathrm{GHz}$," IEEE Trans. Antennas Propag., vol. 61, no. 4, pp. 1876-1888, Apr. 2013.

[16] A.R. Guraliuc, M. Zhadobov, G. Valerio, N. Chahat, and R. Sauleau, "Effect of textile on the propagation along the body at $60 \mathrm{GHz}$," IEEE Trans. Antennas Propag., vol. 62, no. 3, pp. 1489-1494, Mar. 2014.

[17] A.R. Guraliuc, M. Zhadobov, G. Valerio, and R. Sauleau, "Enhancement of on-body propagation at $60 \mathrm{GHz}$ using electro textiles," IEEE Antennas Wireless Propag. Lett., vol.13, pp. 603-606, Mar. 2014.

[18] S. Gabriel, R. W. Lau, and C. Gabriel, "The dielectric properties of biological tissues: III. Parametric models for the dielectric spectrum of tissues," Phys. Med. Biol., vol. 41, no. 11, pp. 2271-2293, Nov. 1996.

[19] A.R. Guraliuc, M. Zhadobov, O. De Sagazan, and R. Sauleau, "Solid phantom for body-centric propagation measurements at $60 \mathrm{GHz}$ ", IEEE Microw. Theory Techn., vol. 62, no. 6, pp. 1373-1380, June 2014. 